\title{
COMPARISON OF HIGHER-ORDER ABERRATION VALUES BETWEEN DIFFERENT ABERROMETRY TECHNOLOGIES IN PREOPERATIVE LASER REFRACTIVE SURGERY PATIENTS
}

\author{
Md Muziman Syah ${ }^{1}$, Khairidzan Mohd Kamal ${ }^{2}$, Najwa Munirah Mohd Yusof ${ }^{1}$, Aminul \\ Hakim Sofian Sauri ${ }^{1}$, Noor Atikah Mad ${ }^{1}$ and Nurul Hana ${ }^{1}$ \\ ${ }^{1}$ Department of Optometry and Visual Science, Kulliyyah of Allied Health Sciences, \\ International Islamic University Malaysia, ${ }^{2}$ Department of Ophthalmology, Kulliyyah of \\ Medicine, International Islamic University Malaysia \\ Presenter: Najwa Munirah Mohd Yusof, najwamunirah235@gmail.com
}

Introduction: Purpose of this study was to evaluate the inter-device agreement of ocular and corneal higher-order aberration measurements using various aberrometers.

Materials and method: Ocular and corneal higher-order aberrations were measured from 57 eyes. For ocular aberration, the measurements were obtained from WASCA Analyzer (Carl Zeiss Meditec, Jena, Germany), i-Profiler Plus (Carl Zeiss Meditec, Jena, Germany) and OPD-Scan III (NIDEK Co. Ltd., Tokyo, Japan). Whereas for corneal aberration, an additional measurement was obtained from Atlas Corneal Topographer 9000 (Carl Zeiss Meditec, Jena, Germany). All the measurements were displayed by the Zernike algorithm. Root mean squares of total, 3rd and 4th order of higher-order aberrations were compared between any three devices using repeated measured analysis of variance. The Bland-Altman analysis and Pearson's correlation were employed to assess agreement and strength of relationship between measurements.

Results: For ocular aberrations, there were no significant difference $(P>0.05)$ in all comparisons except for total higher-order aberration, oblique and horizontal trefoil. Whereas for corneal aberrations, vertical coma, spherical aberration and with-therule/against-the-rule secondary astigmatism between devices were found to be significantly different $(P<0.05)$. The most agreeable device pairs for ocular and corneal aberrations were demonstrated by i-Profiler Plus - OPD Scan III (95\% limit of agreement: -0.340 to 0.730 ) and Atlas Corneal Topographer 9000 - i-Profiler Plus (limit of agreement: 0.196 to 0.520 ) respectively. The $r$-values are stronger than 0.70 .

Conclusion: There were differences in the value of higher-order aberrations between different aberrometry technologies. Clinical impact due to the differences in wavefront measurement between skiascopy, Placido-based and laser technologies need to be investigated further. 